\title{
Characteristics of ultrafast passively mode-locking soliton fiber laser utilizing higher-order mode fibers (Withdrawal Notice)
}

\section{Yi Weng, Zhongqi Pan}

Yi Weng, Zhongqi Pan, "Characteristics of ultrafast passively mode-locking soliton fiber laser utilizing higher-order mode fibers (Withdrawal Notice)," Proc. SPIE 10098, Physics and Simulation of Optoelectronic Devices XXV, 100980E (22 February 2017); doi: 10.1117/12.2251475 


\section{Characteristics of ultrafast passively mode-locking soliton fiber laser utilizing higher-order mode fibers ( withdrawal notice)}

Yi Weng, and Zhongqi Pan

University of Louisiana at Lafayette (United States)

Proc. SPIE 10098, 100980E (2017)

Online Publication Date: 22 February 2017

Withdrawn from Publication: 15 September 2017

Conference Date: 30 J anuary-2 February 2017

Conference Location: San Francisco, California, United States

Conference Title: Physics and Simulation of Optoelectronic Devices XXV

Conference Chairs: Bernd Witzigmann, Marek Osinski, Yasuihiko Arakawa

Publisher's Note: This paper, originally published on 22 February 2017, was withdrawn per author request. 